\title{
Validation comparée de 3 modèles de digestion ruminale pour prédire les flux azotés duodénaux microbiens
}

\author{
B Ramangasoavina, D Sauvant \\ INRA, station de Nutrition et Alimentation, Institut national agronomique Paris-Grignon, \\ 16, rue Claude-Bernard, 75231 Paris Cedex 05, France
}

\begin{abstract}
Summary - Comparative validation of 3 models of ruminal digestion to predict the duodenal $\mathbf{N}$ microbial flow. Three models of ruminal digestion (INRA, Cornell, Baldwin) were validated on a database of 80 measurements of duodenal flow in dairy cows. The 3 models enabled the total duodenal flow of proteins to be predicted fairly satisfactorily; however, they were less sensitive to variations in microbial protein flow.
\end{abstract}

De nombreux modèles ont été publiés à ce jour, pour traduire la digestion réticuloruminale de l'azote. Un travail de validation comparative a été entrepris pour 3 d'entre eux, celui du système PDI (Vérité et al, 1987) du système de Cornell - CO (Fox et $a /, 1988)$ et le modèle mécaniste de Baldwin et al - BA (1987).

Matériel et méthodes — La base de validation est constituée de 80 mesures, issues de 17 publications de la littérature, de flux duodénaux d'azote microbien et total réalisées sur des vaches laitières. Pour 49 de ces bilans, issus de 14 publications, des mesures de flux duodénal de matière organique (MO) ont également été effectuées. Les caractéristiques chimiques et les ingrédients des rations ont été utilisés pour prédire, selon la méthode propre à chacun des 3 modèles, les valeurs des flux duodénaux d'azote total et microbien.

Résultats et discussion - Les valeurs moyennes (minimum-maximum) de la matière sèche ingérée (MSI), de la proportion de fourrage $(F)$ et du taux de matière azotées du régime (TA) ont été respectivement de $14,9(6,4-24,2) \mathrm{kg} / \mathrm{j}, 46,7(10,0-$ $80,0) \%$ et $16,7(8,7-26,8) \%$. Pour les flux duodénaux totaux ( $\mathrm{Nt} \times 6,25$ ) et microbiens $(\mathrm{Nm} \times 6,25)$, ces valeurs sont de 2 $031(530-3800) \mathrm{g} / \mathrm{j}$ et $1063(160-1820) \mathrm{g} /$ j. Pour le sous-groupe de 49 mesures, les caractéristiques précédentes ne diffèrent pas significativement; pour le rendement de la protéosynthèse (RDM, g Nm/kg MO digestible dans le rumen), ces valeurs sont de $27,2(11,7-44,2)$.

Les valeurs prédites et observées des flux d'Nt $\times 6,25$ s'alignent autour de la $1^{\text {re }}$ bissectrice, les coefficents de régression et écarts type résiduels (ETR) sont égaux à 1,00 et $187 \mathrm{~g}, 0,95$ et $294,0,99$ et $250 \mathrm{~g}$ pour les modèles $\mathrm{PDI}, \mathrm{CO}$ et $\mathrm{BA}$ respectivement. Par contre, les 3 modèles ne traduisent que partiellement les variations mesurées du flux des protéines microbiennes. En effet, un accroissement mesuré de $100 \mathrm{~g}$ de protéines microbiennes correspond à des augmentations de 45,43 et $30 \mathrm{~g}$, avec les modèles PDI, $\mathrm{CO}$ et $\mathrm{BA}$, les ETR étant de 113, 193 et 93 respectivement (fig 1a, b, c). Les mêmes tendances apparaissent pour l'analyse inter et intraexpérience des résultats. Pour les 49 bilans, l'étude des facteurs susceptibles d'expliquer le biais (mesuré-prédit) montre que l'efficacité mesurée de la protéosynthèse microbienne était le paramètre le plus important avec des corrélations $(P<0,01)$ de $0,58,0,46$ et 0,71 pour $\mathrm{PDI}, \mathrm{CO}$ et $\mathrm{BA}$ respectivement. Pour les mêmes données, les variations intraexpérience du rendement ont été significativement expliquées par les variations $M S I, F$ et F2, qui sont des paramètres déterminants de l'écosys- 
tème ruminal et dont les effets spécifiques ne sont pas pris en compte dans les 3 modèles considérés.

$$
\begin{gathered}
R D M=\begin{array}{c}
0,97 M S I \\
(0,25)
\end{array} \quad\left(0,39 F-0,0028 F^{2}\right. \\
\left(n=49, R^{2}=0,93, E T R=2,67\right) .
\end{gathered}
$$

En conclusion, ces biais de prédiction mettent en évidence la nécessité de mieux connaître les causes de variation biologiques et éventuellement méthodologiques de l'efficacité de la croissance microbienne pour les intégrer dans les systèmes de prévision des flux d'acides aminés absorbés.

Baldwin RL, Thornley JHM, Beever DE (1987) $J$ Dairy Res 54,107-131

Fox DG, Sniffen CJ, O'Connor JD, Russel JB, Van Soest PJ (1988) Cornell Univ Agric Exp Stat Bull, $72 p$

Vérité R, Chapoutot $P$, Michalet-Doreau B, Peyraud JL, Poncet $C$ (1987) Bull Techn CRZVTheix-INRA, 70, 19-34
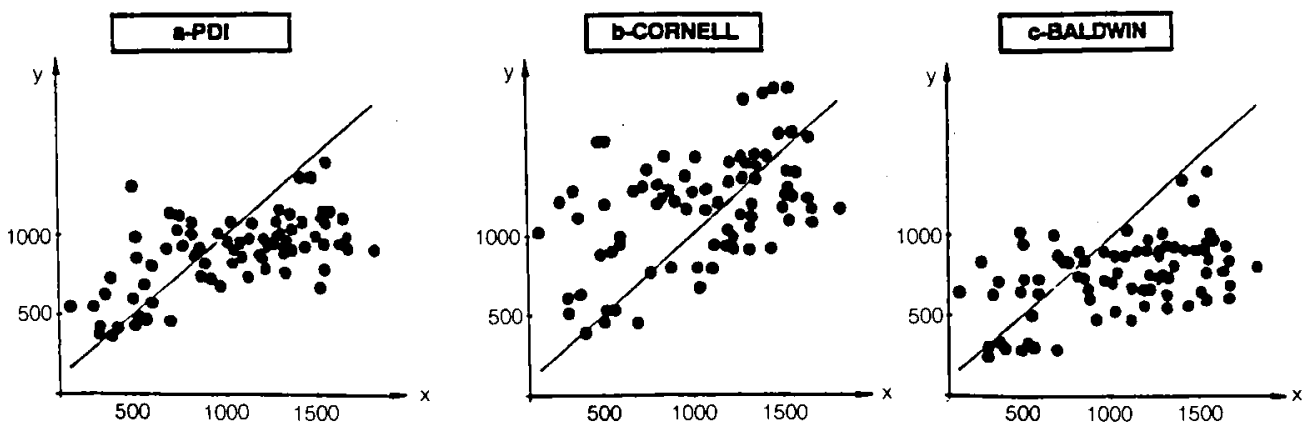

Fig 1. Comparaison entre les valeurs mesurées $(x)$ et prédites par les modèles $(y)$ des matières azotées microbiennes $(g / l)$. 